\title{
Quantum synchronization as a local signature of super- and subradiance
}

\author{
B. Bellomo, ${ }^{1}$ G. L. Giorgi, ${ }^{2}$ G. M. Palma, ${ }^{3}$ and R. Zambrini ${ }^{2}$ \\ ${ }^{1}$ Institut UTINAM - UMR 6213, CNRS, Observatoire des Sciences de l'Univers THETA, Université Bourgogne Franche-Comté, F-25000 \\ Besançon, France \\ ${ }^{2}$ IFISC (UIB-CSIC), Instituto de Física Interdisciplinar y Sistemas Complejos (Universitat de les Illes Balears-Consejo Superior de \\ Investigaciones Científicas), UIB Campus, E-07122 Palma de Mallorca, Spain \\ ${ }^{3}$ NEST, Istituto Nanoscienze Consiglio Nazionale delle Ricerche and Dipartimento di Fisica e Chimica, Università degli Studi di Palermo, Via \\ Archirafi 36, I-90123 Palermo, Italy
}

(Received 21 December 2016; published 7 April 2017)

\begin{abstract}
We study the relationship between the collective phenomena of super- and subradiance and spontaneous synchronization of quantum systems. To this aim we revisit the case of two detuned qubits interacting through a pure dissipative bosonic environment, which contains the minimal ingredients for our analysis. By using the Liouville formalism, we are able to find analytically the ultimate connection between these phenomena. We find that dynamical synchronization is due to the presence of longstanding coherence between the ground state of the system and the subradiant state. We finally show that, under pure dissipation, the emergence of spontaneous synchronization and of subradiant emission occur on the same time scale. This reciprocity is broken in the presence of dephasing noise.
\end{abstract}

DOI: 10.1103/PhysRevA.95.043807

\section{INTRODUCTION}

Superradiance, the collective emission of a sample of atoms, is one of the most celebrated examples of cooperative quantum phenomena, while synchronization is a widespread and paradigmatic emergent behavior in complex systems (see [1] and [2], respectively, for comprehensive reviews). In the most idealized form of superradiance, as first described by Dicke in his seminal paper [3], the atoms identically interact with the electromagnetic field, a situation encountered, e.g., when these are confined in a region of space smaller than the wavelength of the resonant modes. When the initial atomic state is a highly entangled symmetric state, the emission is cooperative and takes place in a rapid burst at a rate proportional to the square of the number of atoms. This is a clear signature of the presence of constructive quantum interference. If the emission starts from the state in which all the atoms are in their excited state, the collective atomic state acts as an antenna amplifying at a macroscopic level the quantum field fluctuation that triggers the emission.

Superradiance in the presence of a small number of emitters [4] has been observed by employing trapped ions [5,6] and superconducting qubits [7]. Superradiant emission has been shown to be a useful resource towards the realization of singlephoton sources [8], quantum memories [9], and laser cooling [10]. In particular, one-dimensional quantum electrodynamics systems are very promising to observe superradiant emission, as the interaction can be maintained over large distances $[7,11,12]$. Furthermore, such a flexible setup makes it possible to explore different coupling regimes and then to study the transition from the case of independent emitters to the collective-decay limit. The counterpart of superradiance, that is, subradiance, is significantly harder to observe experimentally, as subradiant states take place over longer time scales and require protection from any possible source of local noise. It was also experimentally observed in the case of a pair of emitters [5] and more recently in a large system of resonant scatterers in an extended and dilute cold-atom sample [13].
The aim of this paper is to discuss the connection of superradiance with spontaneous synchronization, a widespread phenomenon of adjustment of the dynamical rhythms of different interacting systems [2]. This emergent phenomenon has been largely studied in classical regimes and multidisciplinary contexts. It has been predicted in the quantum regime, in several platforms including harmonic networks [14], mechanical resonators [15], and atomic and ionic setups [16-20]. Different approaches have been considered to frame quantum synchronization referring to both local and global, e.g., quantum correlation, measures (see [21] and references therein). For the purpose of this work, we address the collective quantum phenomenon of super- and subradiance to establish a connection with spontaneous synchronization between the local dynamics of the atoms; we therefore consider spontaneous synchronization as the adjustment to a common rhythm of local observables as in Refs. [18,20].

We mention that both synchronization and superradiance have also been considered in the presence of external driving. Driven synchronization, or entrainment, in the quantum regime has been considered, for instance, in Ref. [22]. On the other hand, superradiance in the presence of driving has been identified as lasing with an extremely narrow linewidth [23], with a collective emission with an extremely high degree of phase coherence [24] and quantum phase locking [25]. It can be observed in general in incoherently pumped arrays of strongly interacting atomic quantum dipoles [19]. Indeed, the emission takes place in rapid bursts with large fluctuations in the emission delay time among the various experimental observations [26]. Further features in the superradiant emissions that are a signature of synchronization take place when the emission is due to a sample of two different detuned atomic species $[27,28]$.

The aim of this work is to consider both superradiance and spontaneous synchronization taking place during dynamical relaxation into a common environment, in the absence of external driving and in the presence of detuning between the atoms. In order to get a full analytic treatment, we consider 
within the Liouville formalism the case of two detuned two-level systems in the presence of dissipation induced by a thermal environment, displaying both superradiant and subradiant emission and dynamical synchronization. We note that, although collective effects are more prominent in the presence of a large number of emitters, most features are already present in the decay of just two atoms coupled via dipole interactions [29-31]. We will show that, in the presence of pure dissipation, the two phenomena are different manifestations of a sole property of the model. Indeed, dynamical synchronization is due to the presence of coherence between the ground state and the subradiant state. Thus, this kind of synchronization has a pure quantum origin, as it directly relies on quantum interference.

The paper is organized as follows. In Sec. II we introduce the model and the master equation used to solve the dynamics. In Sec. III the evolution of the system is studied by employing the so-called Liouville representation for the density matrix. In Sec. IV we introduce quantum synchronization and quantify it in our model. Super- and subradiance are studied in Sec. V and compared to synchronization. The case of purely dephasing noise is the subject of Sec. VI. Finally, a summary is given in Sec. VII.

\section{MODEL}

Our system $S$ consists of two atoms that interact with a common bosonic environment $E$. We will show that this model, already considered in several works [32,33], allows for a fully analytical description of the origin of the connection between the phenomena of synchronization and of super- and subradiance. The two atoms (described by qubits 1 and 2) have different frequencies $\omega_{1}$ and $\omega_{2}$ and their free Hamiltonian is $(\hbar=1)$

$$
H_{S}=\frac{\omega_{1}}{2} \sigma_{1}^{z}+\frac{\omega_{2}}{2} \sigma_{2}^{z},
$$

where $\sigma_{1,2}^{z}=|e\rangle\langle e|-| g\rangle\langle g|$ and $|g\rangle$ and $|e\rangle$ indicate, respectively, the ground and excited states of each atom. The eigenstates of the free Hamiltonian $H_{S}$ form the so-called decoupled basis $\{|e e\rangle,|e g\rangle,|g e\rangle,|g g\rangle\}$ with corresponding energies $\left\{\omega_{0}, \delta,-\delta,-\omega_{0}\right\}$, where $\omega_{0}=\left(\omega_{1}+\omega_{2}\right) / 2$ is the average frequency and $\delta=\omega_{1}-\omega_{2}$ is the detuning between atoms.

The total Hamiltonian of the atoms interacting with the environment is $H_{T}=H_{S}+H_{E}+H_{I}$, where $H_{E}=\sum_{k} \omega_{k} a_{k}^{\dagger} a_{k}$ corresponds to the radiation environment and $H_{I}=-\sum_{i} \hat{\mathbf{d}}_{i}$. $\mathbf{E}_{i}$ is the interaction Hamiltonian between the two atoms $\left(\hat{\mathbf{d}}_{i}=\mathbf{d}_{i} \sigma_{i}^{x}\right.$, with $\mathbf{d}_{i}=\left\langle e\left|\hat{\mathbf{d}}_{i}\right| g\right\rangle$, is the dipole operator of atom $i$ th) and the environmental field $\mathbf{E}_{i}=\sum_{k} \mathcal{E}_{k}^{i}\left(a_{k}+a_{k}^{\dagger}\right)$. The interaction Hamiltonian can be thus put in the form [32]

$$
H_{I}=\sum_{i} \sigma_{i}^{x} \sum_{k} g_{k}^{i}\left(a_{k}+a_{k}^{\dagger}\right)
$$

where $g_{k}^{i}=-\mathbf{d}_{i} \cdot \mathcal{E}_{k}^{i}$.

\section{Master equation}

In the limit of weak system-bath coupling and for zero temperature, the dynamics of the reduced density matrix of the two atoms $\rho$ can be studied by performing the Born-Markov and the secular approximations, noting that, in the limit of small detuning $\delta \ll \omega_{0}$, also terms oscillating with frequency $\omega_{1}-\omega_{2}$ must be kept. The master equation reads [32,34]

$$
\dot{\rho}=-i\left[H_{S}+H_{\mathrm{LS}}, \rho\right]+\sum_{i j} \gamma_{i j}\left(\sigma_{i}^{-} \rho \sigma_{j}^{+}-\frac{1}{2}\left\{\sigma_{j}^{+} \sigma_{i}^{-}, \rho\right\}\right) \text {, }
$$

where $\sigma_{i}^{+}=|e\rangle\langle g|$ and $\sigma_{i}^{-}=|g\rangle\langle e|$. In the following we use the notation $\gamma_{i i} \equiv \gamma_{i}$ and define $\gamma_{0}=\left(\gamma_{1}+\gamma_{2}\right) / 2$, while $\gamma_{12}=$ $\gamma_{21}$ is assumed to be real (its maximal absolute value is given by $\left.\sqrt{\gamma_{1} \gamma_{2}}\right)$. The Lamb-shift Hamiltonian is

$$
H_{\mathrm{LS}}=s_{1} \sigma_{1}^{z}+s_{2} \sigma_{2}^{z}+s_{12}\left(\sigma_{1}^{-} \sigma_{2}^{+}+\sigma_{1}^{+} \sigma_{2}^{-}\right) .
$$

The local shifts $s_{i}$ lead to irrelevant renormalization of the natural frequencies $\omega_{1}$ and $\omega_{2}$, and $s_{12}$ is assumed to be real.

The different parameters appearing in the master equation are directly connected to the correlations functions of the environment and thus to its spectral density. In particular, the damping coefficients $\gamma_{i j}$ depend on the interaction Hamiltonian parameters and on the atomic transition frequencies. Their explicit form in the vacuum electromagnetic field can be found, for instance, in Eqs. (31)-(33) of Ref. [31]. Different physical configurations correspond to different regimes in the parameter space. As an example, a crucial factor to determine whether or not the super- and subradiant regime is achieved is the distance between the atoms. For large separations, the coupling terms $\gamma_{i j}$, with $i \neq j$, are negligible and start being relevant when the separation is of the same order of magnitude as the resonant wavelength. These parameters attain their maximal values in the small-sample limit. Henceforth, in order to explore different regimes, we assume to be able to tune the values of these parameters freely.

\section{DENSITY-MATRIX EVOLUTION IN THE LIOUVILLE REPRESENTATION}

The dynamics of the system can be studied by adopting the so-called Liouville representation of the density matrix. In this representation, the master equation (3) is mapped into a set of linearly coupled differential equations for the elements of the reduced density matrix of the two atoms $\left.\left.\left|\dot{\rho}_{t}\right\rangle\right\rangle=\mathcal{L}\left|\rho_{t}\right\rangle\right\rangle$, where $\left.\left|\rho_{t}\right\rangle\right\rangle$ is a vector in the Hilbert-Schmidt space $\mathcal{H}=\mathbb{C}^{16}$ :

$$
\left.\left.\rho=\sum_{i, j=1}^{4} \rho_{i j}|i\rangle\langle j|\stackrel{\mathrm{HS}}{\longrightarrow}| \rho\rangle\right\rangle=\sum_{i, j=1}^{4} \rho_{i j}|i j\rangle\right\rangle,
$$

where $|i j\rangle\rangle=|i\rangle \otimes|j\rangle$. The inner product in the HilbertSchmidt space is defined by $\langle\langle\tau \mid \rho\rangle\rangle=\operatorname{Tr}\left(\tau^{\dagger} \rho\right)$.

As shown in the Appendix, the total Liouvillian $\mathcal{L}$ turns out to be the direct sum of five terms $\mathcal{L}=\bigoplus_{\mu} \mathcal{L}_{\mu}, \mu \in$ $\{a, b, c, d, e\}$. Thus, $\mathcal{H}$ can be decomposed into five independent subspaces $\mathcal{H}=\bigoplus_{\mu} \mathcal{H}_{\mu}$ whose dimensions are 6, 4, 4, 1, and 1. The form of each $\mathcal{L}_{\mu}$ and the corresponding right $\left.\left(\left|\tau_{i}^{\mu}\right\rangle\right\rangle\right)$ and left $\left(\left\langle\bar{\tau}_{i}^{\mu}\right|\right)$ eigenvectors, together with the eigenvalues, is explicitly reported in the Appendix. We stress that these eigenvectors are not constrained to represent valid states. In fact, as we will see later, the only physical state that is also an eigenvector of $\mathcal{L}$ is the ground state, while the totally excited state is the only physical eigenvector of $\mathcal{L}^{\dagger}$. This implies that, 
for instance, a generic $\left.\left|\tau_{i}^{\mu}\right\rangle\right\rangle$ cannot be taken as the initial state. On the other hand, the dynamics of any density matrix can always be described as a superposition of such nonphysical objects whose linear combination is constrained to be a density matrix.

Within this representation, a key role is played by $\mathcal{L}_{a}$ being $\mathcal{H}_{a}$ generated by the projectors of the four states forming the decoupled basis and by $|e g\rangle\langle g e|$ and $|g e\rangle\langle e g|$. As shown in the Appendix, Sec. 2, the right $(R)$ eigenvectors of $\mathcal{L}_{a}$ [Eq. (A8)] are simply expressed in terms of two nonorthogonal states $\left|S_{R}\right\rangle$ and $\left|A_{R}\right\rangle$, given by

$$
\begin{aligned}
\left|S_{R}\right\rangle & =\frac{\alpha_{S}|e g\rangle+|g e\rangle}{\sqrt{1+\left|\alpha_{S}\right|^{2}}}, \quad \alpha_{S}=\frac{\frac{\gamma_{1}-\gamma_{2}}{2}+i \delta+V}{\gamma_{12}+i 2 s_{12}}, \\
\left|A_{R}\right\rangle & =\frac{\alpha_{A}|e g\rangle+|g e\rangle}{\sqrt{1+\left|\alpha_{A}\right|^{2}}}, \quad \alpha_{A}=\frac{\frac{\gamma_{1}-\gamma_{2}}{2}+i \delta-V}{\gamma_{12}+i 2 s_{12}}
\end{aligned}
$$

for

$$
V=\sqrt{\left(\gamma_{12}+i 2 s_{12}\right)^{2}+\left(\frac{\gamma_{1}-\gamma_{2}}{2}+i \delta\right)^{2}} .
$$

This parameter $V$ characterizes all the decay rates of the system (we use the notation $V_{r}=\operatorname{Re}[V]$ and $V_{i}=\operatorname{Im}[V]$ ) appearing in the eigenvalues of $\mathcal{L}_{a}$ [Eq. (A8)]. In the symmetric configuration, when $\gamma_{1}=\gamma_{2}$ and $\delta=0, V$ simply reduces to $\gamma_{12}+i 2 s_{12}$. On the other hand, the left $(L)$ eigenvectors of $\mathcal{L}_{a}$ [Eq. (A9)] are given in terms of the states

$$
\left|S_{L}\right\rangle=\left|S_{R}\right\rangle^{*}, \quad\left|A_{L}\right\rangle=\left|A_{R}\right\rangle^{*} .
$$

The property $\alpha_{S} \alpha_{A}=-1$ implies that $\left\langle S_{L} \mid A_{R}\right\rangle=\left\langle A_{L} \mid S_{R}\right\rangle=$ 0 .

Notice that, changing $\gamma_{12}$ into $-\gamma_{12}$ (this quantity can also assume negative values [35]), the same spectrum of the Liouvillian is obtained provided that the values of $\gamma_{1}$ and $\gamma_{2}$ are also interchanged. Furthermore, as a reflection of the Liouvillian symmetry, in the case $\gamma_{1}=\gamma_{2}$ and $\delta=0$, the subradiant state switches to the superradiant one and vice versa. On the other hand, if $\gamma_{1}=\gamma_{2}$ but $\delta \neq 0, \gamma_{12} \rightarrow-\gamma_{12}$ implies $\left|S_{R}\right\rangle \rightarrow\left|S_{L}\right\rangle^{*}$ and $\left|S_{L}\right\rangle \rightarrow\left|S_{R}\right\rangle^{*}$.

The states $\left|S_{R}\right\rangle$ and $\left|S_{L}\right\rangle$, and $\left|A_{R}\right\rangle$ and $\left|A_{L}\right\rangle$, appear to be a natural extension of the symmetric $|S\rangle=(|g e\rangle+|e g\rangle) / \sqrt{2}$ and antisymmetric $|A\rangle=(|g e\rangle-|e g\rangle) / \sqrt{2}$ states and they reduce to them in the symmetric configuration $\left(\gamma_{1}=\gamma_{2}\right.$ and $\delta=0)$. Their relevance in the two-qubit dynamics is twofold. On the one hand, $\left|S_{R}\right\rangle$ and $\left|A_{R}\right\rangle$ enter the expression of the right eigenvectors $\left.\left|\tau_{i}^{a}\right\rangle\right\rangle$, so they can naturally be used to get simple expressions for the evolution of the density-matrix operator [see Eq. (8)]. On the other hand, $\left|S_{L}\right\rangle$ and $\left|A_{L}\right\rangle$ enter the expression of the left eigenvectors $\left.\left|\bar{\tau}_{i}^{a}\right\rangle\right\rangle$ [see Eq. (A6)], so the rate equations for the evolution of the populations of the density matrix have the simple form reported in Eq. (A14). This is schematically represented in Fig. 1, which shows that the states $\left|S_{L}\right\rangle$ and $\left|A_{L}\right\rangle$ connect the excited state to the ground state through two independent channels. In particular, the decay rates from $\left|S_{L}\right\rangle$ and $\left|A_{L}\right\rangle$ to the ground state are given, respectively, by $\gamma_{0}+V_{r}$ and $\gamma_{0}-V_{r}$, natural extensions of the decay rates in the symmetric configuration, $\gamma_{0}+\gamma_{12}$ and $\gamma_{0}-\gamma_{12}$. The states $\left|S_{L}\right\rangle$ and $\left|A_{L}\right\rangle$ indeed play a role analogous

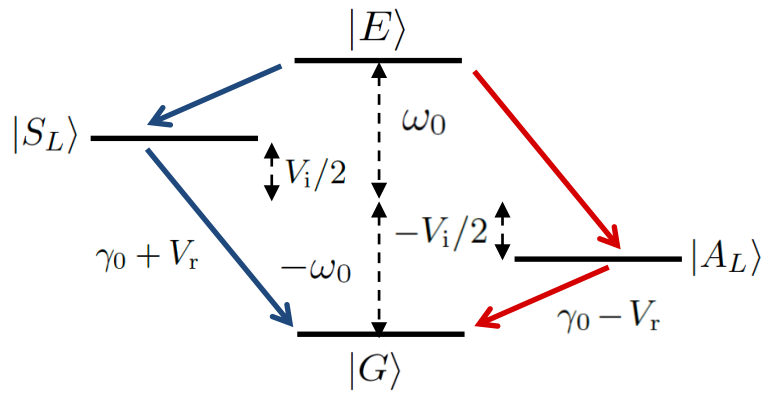

FIG. 1. Schematic representation of two independent channels connecting the excited state $|E\rangle=:|e e\rangle$ (energy equal to $\omega_{0}$ ) to the ground state $|G\rangle=:|g g\rangle$ (energy equal to $-\omega_{0}$ ) [see Eq. (A14)]. The channel passing through the state $\left|S_{L}\right\rangle=\left|S_{R}\right\rangle^{*}$ [whose oscillations are associated with $V_{i} / 2$; see Eq. (A11)] is characterized by a decay rate towards the ground state equal to $\gamma_{0}+V_{r}\left(\gamma_{0}=\frac{\gamma_{1}+\gamma_{2}}{2}\right)$. The channel passing through the state $\left|A_{L}\right\rangle=\left|A_{R}\right\rangle^{*}$ (whose oscillations are associated with $-V_{i} / 2$ ) is characterized by a decay rate towards the ground state equal to $\gamma_{0}-V_{r}$. For simplicity, the channels connecting $|E\rangle$ and $|G\rangle$ through the coherences between $\left|S_{L}\right\rangle$ and $\left|A_{L}\right\rangle$ are not shown.

to the one played by the superradiant state $|S\rangle$ and by the subradiant state $|A\rangle$ in the symmetric case.

We now show that the evolution of the density-matrix operator is simply expressed in terms of the right eigenvectors of $\mathcal{L}$. By using the decomposition for the identity operator in the Hilbert-Schmidt space reported in Eq. (A7) and $\left.\left|\dot{\rho}_{t}\right\rangle\right\rangle=$ $\left.\mathcal{L}\left|\rho_{t}\right\rangle\right\rangle$, one can show that, given an arbitrary initial state $\left.\left|\rho_{0}\right\rangle\right\rangle$, the evolved density matrix can be written (apart from the case of degeneracy in the spectrum of the Liouvillian, as in the case of the Dicke limit when $\gamma_{1}=\gamma_{2}=\gamma_{12}$ and $\delta=0$ ) as

$$
\left.\left.\left|\rho_{t}\right\rangle\right\rangle=\sum_{\mu} \sum_{i} p_{0 i}^{\mu}\left|\tau_{i}^{\mu}\right\rangle\right\rangle e^{\lambda_{i}^{\mu} t}
$$

for $p_{0 i}^{\mu}=\frac{\left\langle\left\langle\bar{\tau}_{i}^{\mu} \mid \rho_{0}\right\rangle\right\rangle}{\left\langle\left\langle\bar{\tau}_{i}^{\mu} \mid \tau_{i}^{\mu}\right\rangle\right\rangle}$, where $\mu$ runs over the five subspaces and $i$ between 1 and $\operatorname{dim}\left(\mathcal{H}_{\mu}\right)$. Equation (8) is the main tool we are going to use in the following sections to make a connection between the phenomena of synchronization and of super- and subradiance.

Before doing that, we consider a couple of examples that will be useful in the rest of this paper. Under particular initial conditions the dynamics is governed by only one decay rate (different from zero). If we start from $\left.\left.\left|\rho_{0}\right\rangle\right\rangle=\left|A_{R} A_{R}\right\rangle\right\rangle$ (i.e., we prepare the system in the pure state $\left|A_{R}\right\rangle$ ), one can see from Eq. (A8) that only two right eigenvectors of $\mathcal{L}_{A}$ contribute to its dynamics, $\left.\left.\left.\left|\rho_{0}\right\rangle\right\rangle=\left|\tau_{1}^{a}\right\rangle\right\rangle+\left|\tau_{6}^{a}\right\rangle\right\rangle$. It follows that, in Eq. (8), $p_{01}^{a}=p_{06}^{a}=1$ (the others $p_{0 i}^{\mu}$ are equal to zero), so at time $t$ we have

$$
\begin{aligned}
\left.\left|\rho_{t}\right\rangle\right\rangle & \left.\left.=\left|\tau_{1}^{a}\right\rangle\right\rangle+\left|\tau_{6}^{a}\right\rangle\right\rangle e^{\lambda_{6}^{a} t} \\
& \left.=|G G\rangle\rangle\left[1-e^{-\left(\gamma_{0}-V_{r}\right) t}\right]+\left|A_{R} A_{R}\right\rangle\right\rangle e^{-\left(\gamma_{0}-V_{r}\right) t} .
\end{aligned}
$$

Equation (9) clearly shows that in this case the vector $\left.\left|A_{R} A_{R}\right\rangle\right\rangle$ is dynamically coupled only to $|G G\rangle\rangle$, its decay rate being given by $\gamma_{0}-V_{r}$. Its role is in this sense analogous to the one played by the subradiant antisymmetric state when $\gamma_{1}=\gamma_{2}$ and $\delta=0$. 
Analogous considerations hold for the vector $\left.\left|S_{R} S_{R}\right\rangle\right\rangle$. If we prepare the system in $\left.\left.\left|\rho_{0}\right\rangle\right\rangle=\left|S_{R} S_{R}\right\rangle\right\rangle$, this implies, using Eq. (A8), that $\left.\left.\left.\left|\rho_{0}\right\rangle\right\rangle=\left|\tau_{1}^{a}\right\rangle\right\rangle+\left|\tau_{5}^{a}\right\rangle\right\rangle$. It follows that $p_{01}^{a}=p_{05}^{a}=1$, so the evolution takes the form

$$
\begin{aligned}
\left.\left|\rho_{t}\right\rangle\right\rangle & \left.\left.=\left|\tau_{1}^{a}\right\rangle\right\rangle+\left|\tau_{5}^{a}\right\rangle\right\rangle e^{\lambda_{5}^{a} t} \\
& \left.=|G G\rangle\rangle\left[1-e^{-\left(\gamma_{0}+V_{r}\right) t}\right]+\left|S_{R} S_{R}\right\rangle\right\rangle e^{-\left(\gamma_{0}+V_{r}\right) t} .
\end{aligned}
$$

The role of the vector $\left.\left|S_{R} S_{R}\right\rangle\right\rangle$ is thus analogous to the one played by the superradiant symmetric state in the case when $\gamma_{1}=\gamma_{2}$ and $\delta=0$.

\section{SYNCHRONIZATION}

This section is devoted to the quantum synchronization phenomenon in our two-qubit system. As we deal with a system relaxing towards a thermal state (at zero temperature, i.e., the ground state), the emergence of synchronization is assessed in a transient (preasymptotic) regime $[14,18]$. We focus on the case of spontaneous synchronization, emerging during the dynamics in the absence of pumping sources at the atoms' locations. Quantum spontaneous synchronization can be quantified through different measures, and local and global indicators and correlations have been used for this purpose, as reviewed in Ref. [21]. A global indicator for quantum synchronization in an atomic context has been considered, for instance, in Ref. [25] and identified as the absence of a relative oscillation in the correlation at different times of two driven-atom clouds. Interestingly, under proper conditions, this correlation can be accessed experimentally through optical measurements.

Here we are interested in comparing the (collective) phenomenon of superradiance with the synchronous evolutions of the atoms' (local) dynamics of either populations or coherences. To this aim, we compute the evolution of the average of arbitrary single-atom operators $O_{k}(k=1,2)$, looking at their respective oscillatory (generally irregular) dynamics. We consider the expectation values $\left\langle O_{1}\right\rangle_{t}$ and $\left\langle O_{2}\right\rangle_{t}$, where $\langle\bullet\rangle_{t}=\operatorname{Tr}\left(\bullet \rho_{t}\right)$ indicates the average of an arbitrary operator $\bullet$ at time $t$. Synchronization between these local observables is quantified through the Pearson correlation coefficient, a widely used measure of the degree of linear dependence between two variables. Given two time-dependent variables $A_{1}$ and $A_{2}$, the Pearson factor $C$ is

$$
C_{A_{1}(t), A_{2}(t)}(\Delta t)=\frac{\int_{t}^{t+\Delta t}\left[A_{1}(t)-\overline{A_{1}}\right]\left[A_{2}(t)-\overline{A_{2}}\right] d t^{\prime}}{\sqrt{\prod_{i=1}^{2} \int_{t}^{t+\Delta t}\left[A_{i}(t)-\bar{A}_{i}\right]^{2} d t^{\prime}}},
$$

where $\bar{A}_{i}=\frac{1}{\Delta t} \int_{t}^{t+\Delta t} A_{i} d t^{\prime}$ and $A_{i}$ are expectation values of quantum operators. This synchronization measure is evaluated in time, considering a sliding window of length $\Delta t$. As a consequence of the definition, $C$ gives a value between +1 and -1 , where $+1(-1)$ indicates the presence of inphase (antiphase) synchronization and 0 the absence of any synchronization. This measure can be generalized to the case of certain time delay between the local dynamics allowing for a phase shift, i.e., embodying a time delay $\delta t$ in one of the two signals within the definition of $C$ as discussed in Ref. [21]. In this way, $C_{A_{1}(t), A_{2}(t+\delta t)}(\Delta t)=+1$ would indicate perfect time-delayed synchronization with delay $\delta t$.
The dynamical study conducted using the Liouville representation allows us to establish how the time scale of the phenomenon of synchronization depends analytically on the physical parameters appearing in the master equation. Any pair of arbitrary local operators $O_{k}, k=1,2$, can be written in the single-atom basis (Bloch representation) $\left\{\sigma_{k}^{x}, \sigma_{k}^{y}, \sigma_{k}^{z}, I_{k}^{d}\right\}$ :

$$
O_{k}=a_{k}^{x} \sigma_{k}^{x}+a_{k}^{y} \sigma_{k}^{y}+a_{k}^{z} \sigma_{k}^{z}+a_{k}^{d} I_{k}^{d} .
$$

By looking at the decomposition of the Liouvillian operator (see the Appendix, Sec. 1), we find that only the right eigenvectors of $\mathcal{L}^{b}$ and $\mathcal{L}^{c}$ contribute to the average of $\sigma_{k}^{x}$ and $\sigma_{k}^{y}$, while only the ones of $\mathcal{L}^{a}$ contribute to the average of $\sigma_{k}^{z}$. It follows that, using Eq. (8), we have

$$
\left\langle O_{k}\right\rangle_{t}=a_{k}^{x}\left\langle\sigma_{k}^{x}\right\rangle_{t}+a_{k}^{y}\left\langle\sigma_{k}^{y}\right\rangle_{t}+a_{k}^{z}\left\langle\sigma_{k}^{z}\right\rangle_{t}+a_{k}^{d},
$$

where

$$
\begin{aligned}
\left\langle\sigma_{k}^{x}\right\rangle_{t} & =\sum_{i} 2\left|p_{0 i}^{b}\left\langle\tau_{i}^{b}\right\rangle_{x k}\right| e^{\operatorname{Re}\left(\lambda_{i}^{b}\right) t} \cos \left[\operatorname{Im}\left(\lambda_{i}^{b}\right) t+\varphi_{i, x k}^{b}\right], \\
\left\langle\sigma_{k}^{y}\right\rangle_{t} & =\sum_{i} 2\left|p_{0 i}^{b}\left\langle\tau_{i}^{b}\right\rangle_{y k}\right| e^{\operatorname{Re}\left(\lambda_{i}^{b}\right) t} \cos \left[\operatorname{Im}\left(\lambda_{i}^{b}\right) t+\varphi_{i, y k}^{b}\right], \\
\left\langle\sigma_{k}^{z}\right\rangle_{t} & =\sum_{i} p_{0 i}^{a}\left\langle\tau_{i}^{a}\right\rangle_{z k} e^{\lambda_{i}^{a} t},
\end{aligned}
$$

with $\left\langle\tau_{i}^{\mu}\right\rangle_{\nu k}=\operatorname{Tr}\left(\sigma_{k}^{\nu} \tau_{i}^{\mu}\right)$ and $\varphi_{i, \nu k}^{b}=\arg \left(p_{0 i}^{b}\left\langle\tau_{i}^{b}\right\rangle_{\nu k}\right)$, for $\nu=$ $x, y, z$, and where we used $\lambda_{i}^{c}=\lambda_{i}^{b *},\left\langle\tau_{i}^{c}\right\rangle_{\nu k}=\left\langle\tau_{i}^{b}\right\rangle_{\nu k}^{*}$, and $p_{0 i}^{c}=p_{0 i}^{b *}$. Here $\tau_{i}^{\mu}$ is the matrix obtained by mapping the eigenstate $\left.\left|\tau_{i}^{\mu}\right\rangle\right\rangle$ back onto the Hilbert space $\left.\left(\tau_{i}^{\mu} \stackrel{\mathrm{HS}}{\longrightarrow}\left|\tau_{i}^{\mu}\right\rangle\right\rangle\right)$. We recall that $\tau_{i}^{\mu}$ is not necessarily a density matrix, while any $\left\langle\sigma_{k}^{v}\right\rangle_{t}$ is well defined at any time.

Equation (A8) implies that only one frequency, equal to $V_{i}$, enters $\left\langle\sigma_{k}^{z}\right\rangle_{t}$. The term oscillating with this frequency decays with a rate $\gamma_{0}$. As for the averages of $\sigma_{k}^{x}$ and $\sigma_{k}^{y}$, each of them contains four oscillating terms. In the worst-case scenario, when all four terms contribute to these averages, the ratios between the instantaneous amplitudes of these oscillating terms are proportional to $e^{\left[\operatorname{Re}\left(\lambda_{i}^{b}\right)-\operatorname{Re}\left(\lambda_{j}^{b}\right)\right] t}$. By using Eq. (A11) one sees that the terms with smallest decay rates are the one with $i=4$ and $i=3$. The long-lasting ratio between the various terms and the one with smallest decay $(i=4)$ decays as $e^{\left[\operatorname{Re}\left(\lambda_{3}^{b}\right)-\operatorname{Re}\left(\lambda_{4}^{b}\right)\right] t}=e^{-V_{r} t}$. In a time scale given by several units of $V_{r}^{-1}$, we expect that only one term still contributes to the averages of $\left\langle\sigma_{k}^{x}\right\rangle_{t}$ and $\left\langle\sigma_{k}^{y}\right\rangle_{t}$. This is the time-scale separation enabling the emergence of spontaneous synchronization $[14,18]$. On the other hand, the decay rate of the oscillating term in $\left\langle\sigma_{k}^{z}\right\rangle_{t}$ is always larger than $V_{r}$. The time scale for the occurrence of synchronization is thus characterized by a parameter $\kappa_{S}$ equal to the real part of $V$, while the frequency of the long-lasting term, giving the frequency of the synchronized oscillations, is related to the imaginary part of $V$ :

$$
\kappa_{S}=V_{r}, \quad \nu_{S}=\omega_{0}-\frac{1}{2} V_{i} .
$$

The real and imaginary parts of $V$ obey $V_{r} V_{i}=2 \gamma_{12} s_{12}+$ $\delta\left(\gamma_{1}-\gamma_{2}\right) / 2$.

The spontaneous emergence of synchronization in a time window is then due to the long lifetime of the eigenmode $\left.\left.\left|\tau_{4}^{b}\right\rangle\right\rangle=\left|A_{R} G\right\rangle\right\rangle$. In other words, the synchronization between 


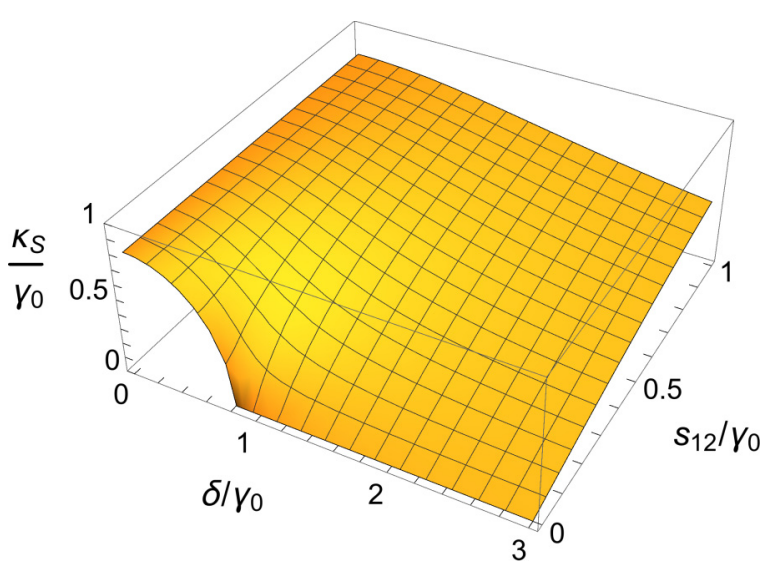

FIG. 2. Plot of $\kappa_{S}$ as a function of $\delta$ and $s_{12}$ in units of $\gamma_{0}$. The values of the other parameters are $\gamma_{1}=\gamma_{2}=\gamma_{0}$ and $\gamma_{12}=0.8 \gamma_{0}$.

averages of arbitrary local operators is due to the presence of longstanding coherences between the ground state and the state $\left|A_{R}\right\rangle$, whose role in the dynamics has been already identified as analogous to the subradiant antisymmetric state. This finding clearly points out in an analytical way the connection between synchronization and subradiant emission, which will be further detailed in the next section. As a side note, we remark that the considerations about the exchange $\gamma_{12} \rightarrow-\gamma_{12}$ made in Sec. III imply that, under such an exchange, the frequency of synchronization would experience a finite jump.

The above considerations allow us to determine the analytical dependence of synchronization on the physical parameters appearing in the master equation. Figure 2 shows the inverse time of synchronization emergence $\kappa_{S}$ depending on $\delta$ and on $s_{12}$, in the case $\gamma_{1}=\gamma_{2}=\gamma_{0}$. In particular, when $s_{12}=0$, $V$ reduces to $\sqrt{\gamma_{12}^{2}-\delta^{2}}$, so for $\delta \geqslant \gamma_{12}, \kappa_{S}$ vanishes and no time-scale separation (synchronization) is found. ${ }^{1}$ Decreasing the atoms' detuning, a sharp transition to synchronization is predicted for $\delta<\gamma_{12}$. This transition to a synchronous dynamics when decreasing the detuning is smooth for $s_{12} \neq 0$, as shown in Fig. 2.

In Fig. 3 the emergence of spontaneous synchronization is shown in the case when the initial state is $\frac{1}{2}(|e\rangle+|g\rangle)(|e\rangle+$ $|g\rangle)$. The plot shows that, during the first stage of the evolution, the dynamics of the averages $\left\langle\sigma_{1}^{x}\right\rangle_{t}$ and $\left\langle\sigma_{2}^{x}\right\rangle_{t}$ are characterized by several frequencies while, after $t \sim \kappa_{S}^{-1}$, only one term oscillating with the frequency $v_{S}$ survives. In the inset, the time evolution of the synchronization factor $C_{\left\langle\sigma_{1}^{x}\right\rangle_{t},\left\langle\sigma_{2}^{x}\right\rangle_{t}}(\Delta t)$ is plotted as a function of time. The dashed curve shows that the two averages $\left\langle\sigma_{1}^{x}\right\rangle_{t}$ and $\left\langle\sigma_{2}^{x}\right\rangle_{t}$ become almost antisynchronized after some units of $\kappa_{S}^{-1}$. This means that while the two signals have become almost monochromatic, their dephasing is close (but not equal) to $180^{\circ}$. The time delayed synchronization $C_{\left\langle\sigma_{1}^{x}\right\rangle_{t},\left\langle\sigma_{2}^{x}\right\rangle_{t+\delta t}}(\Delta t)$ is represented by the solid line. The delay $\delta t$ is the one maximizing the value of $C$.

We remark that it may appear difficult to resolve the fast oscillation and verify the synchronization for values of

\footnotetext{
${ }^{1}$ We stress that in this case and for $\kappa_{S}=0, V_{i}$ is different from zero, leading to two different frequency components in $\left\langle\sigma_{k}^{x}\right\rangle_{t}$ and $\left\langle\sigma_{k}^{y}\right\rangle_{t}$.
}

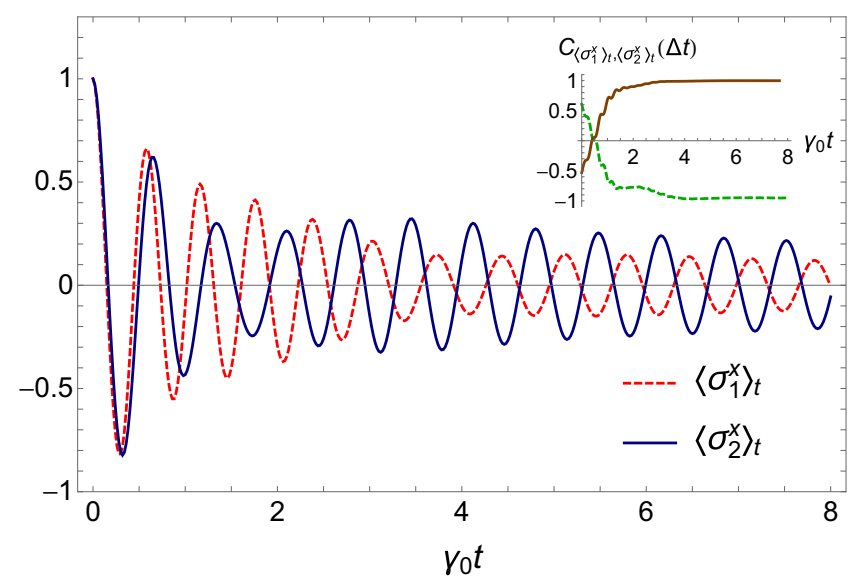

FIG. 3. Time evolution of $\left\langle\sigma_{1}^{x}\right\rangle_{t}$ (dashed line) and $\left\langle\sigma_{2}^{x}\right\rangle_{t}$ (solid line) for the initial state $\frac{1}{2}(|e\rangle+|g\rangle)(|e\rangle+|g\rangle)$. The values of the parameters are $\gamma_{1}=1.1 \gamma_{0}, \gamma_{2}=0.9 \gamma_{0}, \gamma_{12}=0.95 \gamma_{0}, s_{12}=0.6 \gamma_{0}$, $\delta=\gamma_{0}$, and $\omega_{0}=10 \gamma_{0}$. In this case $\kappa_{S}^{-1} \gamma_{0} \approx 1.2$, while the frequency of synchronization is $v_{S}=9.26 \gamma_{0}$. In the inset we plot the evolution of the synchronization measure $C_{\left\langle\sigma_{1}^{x}\right\rangle_{t},\left\langle\sigma_{2}^{x}\right\rangle_{t}}(\Delta t)$ for $\Delta t=2 / \gamma_{0}$ ) (dashed curve) and time delayed one (solid line).

$\omega_{0} \gg \gamma_{0}$. This is the typical situation encountered in ultracold atoms. In this scenario the synchronization could be observed via homodyne and interferometric detection, which allows for phase detection, getting rid of the fast oscillation at the atomic transition frequency. On the other hand, there also exist different setups where superradiance has been experimentally observed in the presence of relatively strong decay rates. For instance, in Ref. [11] two transmon qubits coupled to a single coplanar waveguide resonator were considered in the strong cavity-atom coupling regime, where radiative terms are much larger than any other competing noise effect.

As discussed at the end of Sec. III, there are particular choices of the initial state for which only a frequency is involved in the dynamics giving rise to synchronous dynamics from the initial time, as we show in Fig. 4. Figures 4(a) and 4(b) refer, respectively, to $\frac{1}{\sqrt{2}}\left(|G\rangle+\left|A_{R}\right\rangle\right)$ and to $\frac{1}{\sqrt{2}}\left(|G\rangle+\left|S_{R}\right\rangle\right)$. In both cases, by using Eqs. (A8), (A12), and (13) it is easy to see that only one term is present in the averages of $\left\langle\sigma_{1}^{x}\right\rangle_{t}$ and $\left\langle\sigma_{2}^{x}\right\rangle_{t}$. In Fig. 4(a) the only frequency present is related to $\left.\left|\tau_{4}^{b}\right\rangle\right\rangle$, while in Fig. 4(b) only $\left.\left|\tau_{3}^{b}\right\rangle\right\rangle$ contributes. The two signals are synchronized from the beginning, their decay rates and the relative phase between the two averages (time delay) being different. In particular, starting from the initial state $\frac{1}{\sqrt{2}}\left(|G\rangle+\left|A_{R}\right\rangle\right)$, the two averages end up being equal to

$$
\begin{aligned}
\left\langle\sigma_{1}^{x}\right\rangle_{t} & =\frac{\left|\alpha_{A}\right| e^{-\left(\gamma_{0}-V_{r}\right) t / 2}}{\sqrt{1+\left|\alpha_{A}\right|^{2}}} \cos \left[v_{S} t-\arg \left(\alpha_{A}\right)\right], \\
\left\langle\sigma_{2}^{x}\right\rangle_{t} & =\frac{e^{-\left(\gamma_{0}-V_{r}\right) t / 2}}{\sqrt{1+\left|\alpha_{A}\right|^{2}}} \cos \left[v_{S} t\right] .
\end{aligned}
$$

The relative delay of the two synchronized signals is then equal to $\arg \left(\alpha_{A}\right)$ [in Fig. 4(a) this is $\approx 162^{\circ}$ ]. 

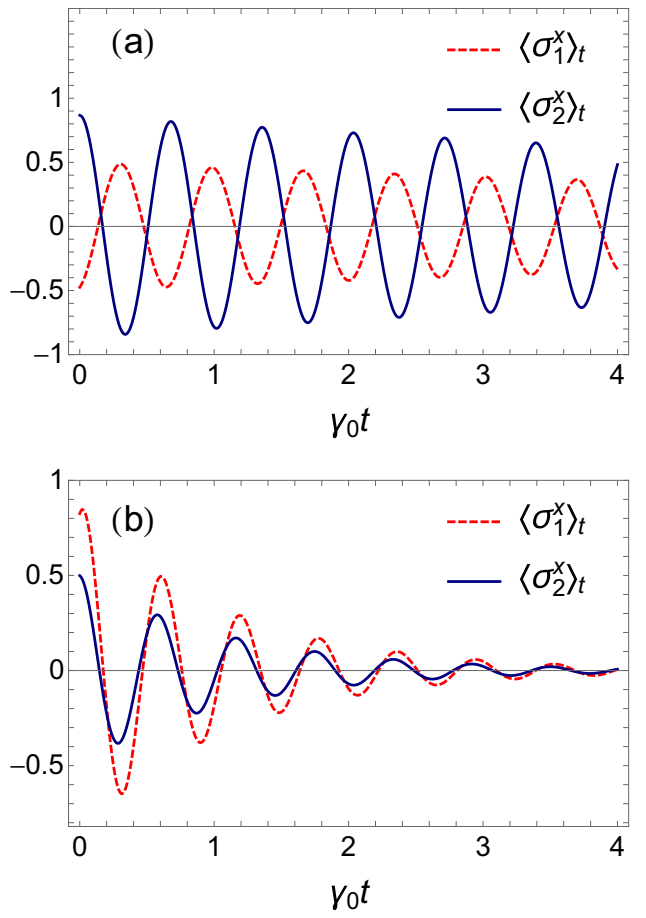

FIG. 4. Time evolution of $\left\langle\sigma_{1}^{x}\right\rangle_{t}$ (dashed line) and $\left\langle\sigma_{2}^{x}\right\rangle_{t}$ (solid line). Time is scaled with $\gamma_{0}$. The initial state is (a) $\frac{1}{\sqrt{2}}\left(|G\rangle+\left|A_{R}\right\rangle\right)$ and (b) $\frac{1}{\sqrt{2}}\left(|G\rangle+\left|S_{R}\right\rangle\right)$. The parameters have the same values as in Fig. 3.

Analogously, when the initial state is $\frac{1}{\sqrt{2}}\left(|G\rangle+\left|S_{R}\right\rangle\right)$, the two averages are given by

$$
\begin{aligned}
\left\langle\sigma_{1}^{x}\right\rangle_{t}= & \frac{\left|\alpha_{S}\right| e^{-\left(\gamma_{0}+V_{r}\right) t / 2}}{\sqrt{1+\left|\alpha_{S}\right|^{2}}} \\
& \times \cos \left[\left(\omega_{0}+\frac{1}{2} V_{i}\right) t-\arg \left(\alpha_{S}\right)\right], \\
\left\langle\sigma_{2}^{x}\right\rangle_{t}= & \frac{e^{-\left(\gamma_{0}-V_{r}\right) t / 2}}{\sqrt{1+\left|\alpha_{S}\right|^{2}}} \cos \left[\left(\omega_{0}+\frac{1}{2} V_{i}\right) t\right] .
\end{aligned}
$$

The relative delay of the two averages is now equal to $\arg \left(\alpha_{S}\right)$ [in Fig. 4(b), this is $\approx 18^{\circ}$ ].

The value of the coefficient $\gamma_{12}$ can be taken as a measure of the transition between the common-bath $\left(\gamma_{12}=\sqrt{\gamma_{1} \gamma_{2}}\right)$ and the separate-bath $\left(\gamma_{12}=0\right)$ cases. The transition between these two cases is discussed in Ref. [36]. In the limit of completely independent atoms, we also have $s_{12}=0$. In the case of independent atoms $\left(\gamma_{12}=0\right.$ and $\left.s_{12}=0\right),\left\langle\sigma_{1}^{x}\right\rangle_{t}$ and $\left\langle\sigma_{2}^{x}\right\rangle_{t}$ contain just one term decaying with rates respectively equal to $\gamma_{1}$ and $\gamma_{2}$ and oscillating with frequencies equal to $\omega_{1}$ and $\omega_{2}$. No synchronization is then possible in this limit case.

In our model, it is the coupling with a common environment that is responsible for the terms in Eq. (3) depending on $\gamma_{12}$ and $s_{12}$, causing the spontaneous synchronization described in this section. However, the presence of a common environment is not the only way of achieving synchronization. In fact, from Eq. (6) one can deduce that the roles played by $\gamma_{12}$ and $s_{12}$ are in some sense complementary. A significant direct interaction between the two atoms (present also in the absence of the environment) can compensate for the absence of a common damping $\gamma_{12}$. This kind of mechanism leading to synchronization was extensively discussed in Ref. [20], in the presence of direct coupling between the two atoms and of a local bath, where it was also shown that in such a scenario the absence of detuning can be detrimental. In fact, from Eq. (6) it follows that for $\gamma_{12}=0$ and $\delta=0$, a threshold behavior completely analogous to the one observed in Fig. 2 would occur. Indeed, for any $s_{12} \geqslant\left(\gamma_{1}-\gamma_{2}\right) / 4, \kappa_{S}$ is exactly equal to zero and dynamical synchronization cannot occur. As a matter of fact, in the case of two identical baths and in the absence of detuning, the two atoms are identical and their trajectories can only be different because of the initial condition.

\section{SUPER-AND SUBRADIANCE}

In order to study the occurrence of super- and/or subradiance we compute the evolution of the total radiation rate (given in photons per second) [1]

$$
I(t)=\sum_{i, j} \gamma_{i j}\left\langle\sigma_{i}^{+} \sigma_{j}^{-}\right\rangle_{t},
$$

given by the average of the operator $Q=\sum_{i, j} \gamma_{i j} \sigma_{i}^{+} \sigma_{j}^{-}$. All the matrix elements associated with the operators $\left\langle\sigma_{i}^{+} \sigma_{j}^{-}\right\rangle$exist in the Liouvillian subspace $\mathcal{L}^{a}$. It follows that $I(t)$ is given by

$$
I(t)=\sum_{i} p_{0 i}^{a}\left\langle\tau_{i}^{a}\right\rangle_{Q} e^{\lambda_{i}^{a} t},
$$

where $\left\langle\tau_{i}^{a}\right\rangle_{Q}=\operatorname{Tr}\left(Q \tau_{i}^{a}\right)$. In particular, $\left\langle\tau_{1}^{a}\right\rangle_{Q}=0$ [the ground state does not contribute to $I(t)]$. In the two cases when the initial states are chosen, respectively, as $\left.\left|A_{R} A_{R}\right\rangle\right\rangle$ and $\left.\left|S_{R} S_{R}\right\rangle\right\rangle$, by using Eqs. (9) and (10) one finds

$$
\begin{aligned}
I(t) & =-\lambda_{6}^{a} e^{\lambda_{6}^{a} t}=\left(\gamma_{0}-V_{r}\right) e^{-\left(\gamma_{0}-V_{r}\right) t} \\
\left(\text { for }\left|\rho_{0}\right\rangle\right\rangle & \left.\left.=\left|A_{R} A_{R}\right\rangle\right\rangle\right), \\
I(t) & =-\lambda_{5}^{a} e^{\lambda_{5}^{a} t}=\left(\gamma_{0}+V_{r}\right) e^{-\left(\gamma_{0}+V_{r}\right) t} \\
\left(\text { for }\left|\rho_{0}\right\rangle\right\rangle & \left.\left.=\left|S_{R} S_{R}\right\rangle\right\rangle\right) .
\end{aligned}
$$

Equations (19) describe how the two-atom system emits when initially prepared either in the state $\left|A_{R}\right\rangle$ or in $\left|S_{R}\right\rangle$. Both emissions are characterized by only one decay rate $\gamma_{0}-V_{r}$ and $\gamma_{0}+V_{r}$, respectively, displaying the known different radiances for nonvanishing $V_{r}$. This feature is a first quantitative link with the synchronization parameter $\kappa_{S}=V_{r}$ defined in Eq. (14). When $\kappa_{S}$ increases towards its maximum given by $\gamma_{0}$ (faster synchronization), the decay rates in Eq. (19) go, respectively, to 0 (subradiance) and to $\gamma_{1}+\gamma_{2}$ (superradiance).

Figure 5 shows the evolution of $I(t)$ (on a logarithmic scale) for several initial conditions. One sees that, by starting from $\left|S_{R}\right\rangle$ or $\left|A_{R}\right\rangle$, the decay of $I(t)$ is governed by only one decay rate [see Eq. (19)]. The same holds for the case of independent atoms (IAs) $\left(\gamma_{12}=s_{12}=0\right)$ having individual decay rates equal to $\gamma_{0}$. For any other initial states, the evolution of $I(t)$ is not an exponential function as it depends on more than one decay rate. Starting from the symmetric and antisymmetric states $|S\rangle$ and $|A\rangle$, respectively, the total radiation rates are initially the larger and the smaller among all the possible one-excitation states. However, during the dynamics, part of the population remains trapped in $\left|A_{R}\right\rangle$, whose decay rate 


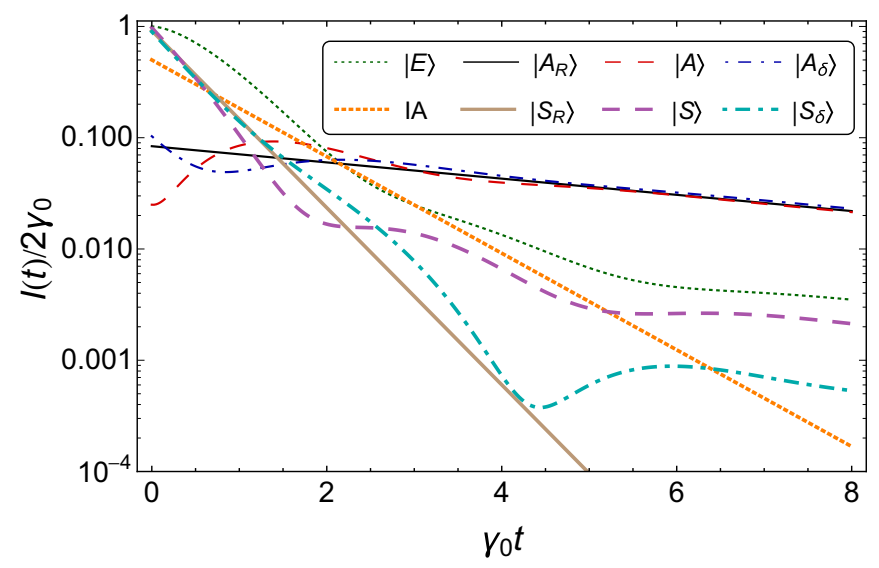

FIG. 5. Time evolution of $I(t) / 2 \gamma_{0}$ (logarithmic scale) for several initial conditions (see the legend; the curves have different styles and thicknesses). Time is scaled with $\gamma_{0}$. The parameters have the same values as in Fig. 3. Here $|S\rangle$ and $|A\rangle$ are the symmetric and antisymmetric states, respectively, and $\left|S_{\delta}\right\rangle$ and $\left|A_{\delta}\right\rangle$ are the oneexcitation states diagonalizing the dressed Hamiltonian $H_{S}+H_{L S}$. The IA case is the one of independent atoms having individual decay rates equal to $\gamma_{0}$, starting from an arbitrary one-excitation state.

(the decay rate of the eigenvector $\left.\left|\tau_{6}^{a}\right\rangle\right\rangle$ ) governs the final part of the evolution of $I(t)$. Similar considerations hold for the initial states $\left|S_{\delta}\right\rangle$ and $\left|A_{\delta}\right\rangle$ [the states that diagonalize the dressed Hamiltonian $H_{S}+H_{\mathrm{LS}}$ of Eq. (3)]. Also the case in which initially both atoms are excited, the condition typically considered in the studies of super- and subradiance, is plotted.

In general (i.e., for most atoms initial states), the large-time behavior of $I(t)$ is governed by the long-lasting eigenvector $I_{\mathrm{sr}}(t)=p_{06}^{a}\left\langle\tau_{6}^{a}\right\rangle_{Q} e^{\lambda_{6}^{a} t}=-p_{06}^{a} \lambda_{6}^{a} e^{\lambda_{6}^{a} t}$, which is the subradiant contribution of $\left.\left|\tau_{6}^{a}\right\rangle\right\rangle$ weighted by the overlap between this eigenmode and the considered initial state. Furthermore, the ratios between the other contributions and the one with the slowest decay all decay faster than $e^{\left(\operatorname{Re}\left[\lambda_{3}^{a}\right]-\lambda_{6}^{a}\right) t}=$ $e^{\left(\operatorname{Re}\left[\lambda_{4}^{a}\right]-\lambda_{6}^{a}\right) t}=e^{-V_{r} t}$, that is, with a characteristic time that is equal to the synchronization time [see Eq. (14)] $\kappa_{S}^{-1}=1 / V_{r}$. This clearly shows how the time scales of synchronization and of subradiant emission are intimately related.

This analysis establishes analytically that the two phenomena under study, that is, synchronization and super- and subradiance, occur at the same time scale. This is displayed in Fig. 6, representing the delayed synchronization measure $C(\Delta t)$ defined in Eq. (11) and a subradiance parameter $R_{I}(t)$, defined as the minimum between $I(t) / I_{\mathrm{sr}}(t)$ and its inverse $I_{\mathrm{sr}}(t) / I(t)$. The parameter $R_{I}(t)$ is such that it goes to 1 when $I(t)$ approaches its large time value $I_{6}(t)$ (from above or below). In addition, $C(\Delta t)$ is computed using in Eq. (11) $\left\langle\sigma_{i}^{x}\right\rangle_{t}$ as local operators and allowing for a time delay between the two signals. The comparison is done by varying $\gamma_{12}$ from 0 to its maximum $\gamma_{0}\left(\gamma_{1}=\gamma_{2}=\gamma_{0}\right.$ in the figure $)$. The above parameters are computed at a time $t^{*}=5 / \gamma_{0}$ such that for low values of $\gamma_{12},\left\langle\sigma_{1}^{x}\right\rangle_{t}$ and $\left\langle\sigma_{2}^{x}\right\rangle_{t}$ are not yet synchronized and $I(t)$ contains still more than one contribution from the eigenstates of $\mathcal{L}^{a}$. By increasing $\gamma_{12}, V_{r}$ increases and we may observe at the chosen time $t^{*}$ the occurrence of synchronization and $I(t)$ approaching its subradiant contribution $I_{\mathrm{sr}}(t)$.

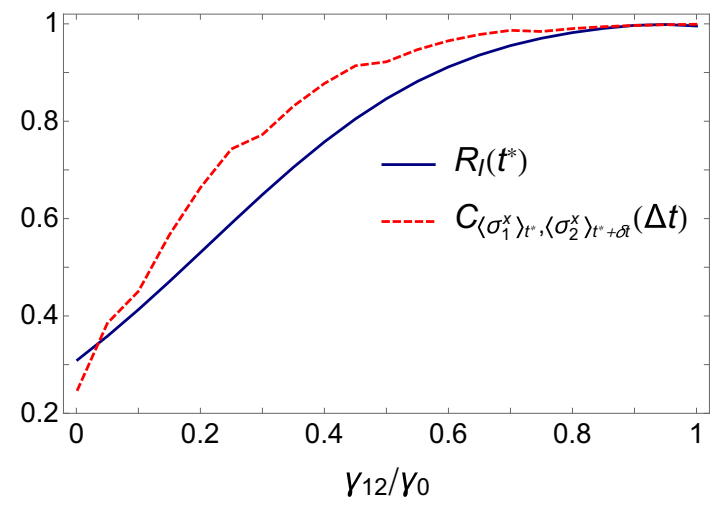

FIG. 6. Comparison between the synchronization measure $C_{\left\langle\sigma_{1}^{x}\right\rangle_{t^{*}},\left\langle\sigma_{2}^{x}\right\rangle_{\left(t^{*}+\delta t\right)}}(\Delta t)\left(\Delta t=2 / \gamma_{0}\right)$ and the ratio $R_{I}\left(t^{*}\right)$, both computed at $t^{*}=5 / \gamma_{0}$ for the initial state $\frac{1}{2}(|e\rangle+|g\rangle)(|e\rangle+|g\rangle)$. The values of the parameters are $\gamma_{1}=\gamma_{2}=\gamma_{0}, s_{12}=0.6 \gamma_{0}, \delta=\gamma_{0}$, and $\omega_{0}=10 \gamma_{0}$. For $\gamma_{12}=\gamma_{0}$, the synchronization parameter is such that $\kappa_{S}^{-1} \gamma_{0} \approx 1.2$, while for $\gamma_{12}=0.3 \gamma_{0}, \kappa_{S}^{-1} \gamma_{0} \approx 4.3$.

\section{DEPHASING NOISE}

So far we have discussed the case of a fully dissipating environment and found a perfect connection between the time scales of emergence of synchronization and superradiance. As discussed in Ref. [18], the presence of decohering noise, even if into a common environment, does not favor synchronization. Here we also show that it alters the interplay with superradiance.

Although the presence of decoherence can be treated exactly [37], in the weak-coupling limit dephasing effects can be included by adding a phenomenological term to the master equation of Eq. (3) [38]:

$$
\mathcal{D}_{\mathrm{dep}}=\sum_{i j} \gamma_{i j}^{d}\left(\sigma_{i}^{z} \rho \sigma_{j}^{z}-\frac{1}{2}\left\{\sigma_{j}^{z} \sigma_{i}^{z}, \rho\right\}\right),
$$

where $\gamma_{21}^{d}=\gamma_{12}^{d}$ is assumed to be real and $\gamma_{12}^{d} \leqslant \sqrt{\gamma_{11}^{d} \gamma_{22}^{d}}$. One can verify that this extra term does not modify the decomposition into the direct sum of sectors of the new Liouvillian. In particular, looking at $\mathcal{L}_{b}$, its new eigenvalues $\lambda_{i}^{b}$ are all equal to the old ones apart from the common term $2\left(\gamma_{11}^{d}+\gamma_{22}^{d}\right)$ that must be subtracted from all of them. It follows that, analogously to what was found in Sec. IV, the time scales for the emergence of quantum synchronization (survival of one term in the average of arbitrary single-atom operators, with respect to the other contributions) remain the same, given by $\kappa_{S}^{-1}=1 / V_{r}$. However, the amplitude of this survival term now is lowered at any time $t$ by a factor $e^{-2\left(\gamma_{11}^{d}+\gamma_{22}^{d}\right) t}$.

As for $\mathcal{L}_{a}$, the situation is different and we can distinguish two main cases. In the case when $\gamma_{11}^{d}+\gamma_{22}^{d}=2 \gamma_{12}^{d}, \mathcal{L}_{a}$ is not modified and our previous analysis remains the same. When this condition is not satisfied, in the general case we do not have analytical expressions for the eigenvalues $\mathcal{L}_{a}$ anymore. However, a numerical analysis shows that the ratio between the exponentials governed by the two smallest (in modulus) decay rates now depends on the value of $\gamma_{11}^{d}+\gamma_{22}^{d}-2 \gamma_{12}^{d}$. This means that in general the time scales of spontaneous 
synchronization and of the emergence of subradiant emission are not equal anymore.

\section{CONCLUSION}

Superradiance is a genuinely collective phenomenon, as it cannot be explained by taking only individual constituents of the whole atomic system. The main question addressed in this paper concerns the possible evidence of such a global effect on the dynamics and synchronization of local observables. In fact, we have found a strict analytical relationship between subradiance and the dynamical synchronization of local trajectories. Considering two detuned qubits coupled to a dissipative bath, by means of the Liouville formalism, we have studied the microscopic mechanisms responsible for the two phenomena and found that, actually, synchronization and super- and subradiance are closely related in our model. As a matter of fact, the synchronization between averages of arbitrary single-atom operators is due to the presence of longstanding coherences between the ground state and a state that can be identified as responsible for subradiant emission, whose experimental detection is known to be more demanding $[5,13]$. The fact that the decay rate due to such a subradiant state is twice the decay rate of the coherence responsible for synchronization clearly shows that the two phenomena are intimately related and occur on the same time scale. Finally, we have shown that by adding the effects of a dephasing noise, the correspondence between synchronization and collective super- and subradiance effects can be altered.

The case of two atoms we have discussed here offers the advantage that a fully analytical treatment is available that makes patent the liaison between subradiance and synchronization. The comparison in the case of an ensemble of $N$ atoms cannot be treated analytically and requires numerical or approximated descriptions, like the mean field adopted, for instance, in Ref. [25] to assess the steady state in the presence of driving. However, in the case of two interacting clouds of different atomic species [1], in some symmetric configurations, one can expect that results similar to the ones obtained here could be valid with the occurrence of synchronization between macroscopic quantities such as the sum of individual spins in each cloud. Indeed, the time evolution of the beating note for sufficient long times should provide a signature of such a phenomenon. On the other hand, the study of the effects of the driving cannot be fully analytical even in the case of only two atoms. In this case open questions remain about the connection between subradiance and synchronization and their relative time scales.

The occurrence of synchronization in classical systems, as well as its definition in a broad spectrum of regimes, is well established [2]. When it comes to the quantum realm, it becomes interesting to identify specific quantum signatures of this effect. Recently, there have been many attempts to quantify the appearance of synchronization also through quantum indicators of (global) correlations [21]. Our work represents a contribution also in this context, given that it offers an explanation of spontaneous synchronization of local observables as the manifestation of collective quantum interference. In other words, the present analytical description through local generic observables dynamics allows us to establish how synchronization witnesses a collective quantum phenomenon of subradiance, representing then a genuine quantum feature of synchronization, intimately different from its classical counterpart.

\section{ACKNOWLEDGMENTS}

B.B. thanks D. Viennot for useful discussions. Funding from EU project QuProCS (Grant Agreement No. 641277), MINECO, AEI/FEDER (Grants No. FIS2014-60343-P and No. FIS2016-78010-P), "Vicerectorat d'Investigació i Postgrau" through the visiting professors program of the UIB and from "Soutien aux enseignants-chercheurs et chercheurs et nouveaux arrivants 2016" of the UFC are acknowledged.

\section{APPENDIX: LIOUVILLE REPRESENTATION}

To treat the dynamics of the system, we adopted in Sec. III the so-called Liouville representation of the density matrix, defined by

$$
\left.\left.\rho=\sum_{i, j=1}^{4} \rho_{i j}|i\rangle\langle j|\stackrel{\mathrm{HS}}{\longrightarrow}| \rho\rangle\right\rangle=\sum_{i, j=1}^{4} \rho_{i j}|i j\rangle\right\rangle,
$$

where $|i j\rangle\rangle=|i\rangle \otimes|j\rangle$. The inner product in the HilbertSchmidt space is defined by $\langle\langle\tau \mid \rho\rangle\rangle=\operatorname{Tr}\left(\tau^{\dagger} \rho\right)$. It follows that for any operator of the system $O$ we have

$$
\left.|O \rho\rangle\rangle=O \otimes \mathbb{I}|\rho\rangle\rangle, \quad|\rho O\rangle\rangle=\mathbb{I} \otimes O^{\top}|\rho\rangle\right\rangle,
$$

where $O^{\top}$ denotes the matrix transposition of $O$ and $\mathbb{I}$ the four-dimensional identity matrix. By using these rules, we obtain the Liouville representation of the master equation of Eq. (3), $|\dot{\rho}\rangle\rangle=\mathcal{L}|\rho\rangle\rangle$, where $\mathcal{L}$ is defined by

$$
\begin{aligned}
\mathcal{L}= & -i\left(H_{\mathrm{eff}} \otimes \mathbb{I}-\mathbb{I} \otimes H_{\mathrm{eff}}^{\top}\right) \\
& +\frac{1}{2} \sum_{i j} \gamma_{i j}\left[2 \sigma_{i}^{-} \otimes\left(\sigma_{j}^{+}\right)^{\top}-\sigma_{j}^{+} \sigma_{i}^{-} \otimes \mathbb{I}-\mathbb{I} \otimes\left(\sigma_{j}^{+} \sigma_{i}^{-}\right)^{\top}\right],
\end{aligned}
$$

where $H_{\text {eff }}=H_{S}+H_{\mathrm{LS}}$. The Liouville representation of the master equation is thus a Schrödinger equation governed by a non-self-adjoint generator $\mathcal{L}^{\dagger} \neq \mathcal{L}$.

\section{Decomposition of $\mathcal{L}$}

By using Eq. (A3), one finds that the total Liouvillian can be decomposed in five Jordan blocks. The Hilbert-Schmidt space $\mathcal{H}=\mathbb{C}^{16}$ can be thus decomposed into five independent subspaces $\mathcal{H}=\bigoplus_{\mu} \mathcal{H}_{\mu}, \mu \in\{a, b, c, d, e\}$, with $\operatorname{dim} \mathcal{H}_{a}=6$, $\operatorname{dim} \mathcal{H}_{b}=\operatorname{dim} \mathcal{H}_{c}=4$, and $\operatorname{dim} \mathcal{H}_{d}=\operatorname{dim} \mathcal{H}_{e}=1$, such that $\mathcal{L}=\bigoplus_{\mu} \mathcal{L}_{\mu}\left(\mathcal{L}_{\mu}\right.$ being an operator of $\left.\mathcal{H}_{\mu}\right)$. Subspace $\mathcal{H}_{a}$ is generated by the basis elements $|e e\rangle\langle e e|| e g\rangle,\langle e g|| e g\rangle,\langle g e|$, $|g e\rangle\langle e g|| g e\rangle,\langle g e|$, and $|g g\rangle\langle g g| ; \mathcal{H}_{b}$ by $|e e\rangle\langle e g|| e e\rangle,\langle g e|$, $|e g\rangle\langle g g|$, and $|g e\rangle\langle g g| ; \mathcal{H}_{c}$ by their complex conjugate counterparts $|e g\rangle\langle e e|| g e\rangle,\langle e e|| g g\rangle,\langle e g|$, and $|g g\rangle\langle g e| ; \mathcal{H}_{d}$ by $|e e\rangle\langle g g|$; and $\mathcal{H}_{e}$ by its conjugate $|g g\rangle\langle e e|$. 
The matrix representations of $\mathcal{L}_{\mu}$ in the basis of $\mathcal{H}_{\mu}$ are given by

$$
\begin{aligned}
\mathcal{L}_{a}=\left(\begin{array}{cccccc}
-\left(\gamma_{1}+\gamma_{2}\right) & 0 & 0 & 0 & 0 & 0 \\
\gamma_{2} & -\gamma_{1} & -\frac{\gamma_{12}}{2}+i s_{12} & -\frac{\gamma_{12}}{2}-i s_{12} & 0 & 0 \\
\gamma_{12} & -\frac{\gamma_{12}}{2}+i s_{12} & -\frac{\gamma_{1}+\gamma_{2}}{2}-i \delta & 0 & -\frac{\gamma_{12}}{2}-i s_{12} & 0 \\
\gamma_{12} & -\frac{\gamma_{12}}{2}-i s_{12} & 0 & -\frac{\gamma_{1}+\gamma_{2}}{2}+i \delta & -\frac{\gamma_{12}}{2}+i s_{12} & 0 \\
\gamma_{1} & 0 & -\frac{\gamma_{12}}{2}-i s_{12} & -\frac{\gamma_{12}}{2}+i s_{12} & -\gamma_{2} & 0 \\
0 & \gamma_{1} & \gamma_{12} & \gamma_{12} & \gamma_{2} & 0
\end{array}\right), \\
\mathcal{L}_{b}=\left(\begin{array}{cccc}
-\gamma_{1}-\frac{\gamma_{2}}{2}-i\left(\omega_{0}-\frac{\delta}{2}\right) \\
-\frac{\gamma_{12}}{2}+i s_{12}
\end{array} \gamma_{12}^{-\frac{\gamma_{1}}{2}-\gamma_{2}-i\left(\omega_{0}+\frac{\delta}{2}\right)}\right. \\
\gamma_{1}
\end{aligned}
$$

while $\mathcal{L}_{c}$ is the conjugate of $\mathcal{L}_{b}, \mathcal{L}_{d}$ has just one element equal to $-\gamma_{0}-2 i \omega_{0}$, and $\mathcal{L}_{e}$ is the conjugate of $\mathcal{L}_{d}$.

\section{Eigenvectors and eigenvalues}

Let $\left\{\lambda_{i}^{\mu}\right\},\left\{\tau_{i}^{\mu}\right\}$, and $\left\{\bar{\tau}_{i}^{\mu}\right\}$ be the eigenvalues and the right and left instantaneous eigenvectors of $\mathcal{L}$. They are defined by

$$
\left.\left.\left.\left.\mathcal{L}\left|\tau_{i}^{\mu}\right\rangle\right\rangle=\lambda_{i}^{\mu}\left|\tau_{i}^{\mu}\right\rangle\right\rangle, \quad \mathcal{L}^{\dagger}\left|\bar{\tau}_{i}^{\mu}\right\rangle\right\rangle=\lambda_{i}^{\mu *}\left|\bar{\tau}_{i}^{\mu}\right\rangle\right\rangle
$$

with

$$
\left\langle\left\langle\bar{\tau}_{i}^{\mu} \mid \tau_{j}^{\nu}\right\rangle\right\rangle=\operatorname{Tr}\left(\bar{\tau}_{i}^{\mu \dagger} \tau_{j}^{\nu}\right)=\left\langle\left\langle\bar{\tau}_{i}^{\mu} \mid \tau_{i}^{\mu}\right\rangle\right| \delta_{\mu \nu} \delta_{i j}
$$

We can write the identity in the Hilbert-Schmidt space $\mathcal{I}$ by means of the right and left instantaneous eigenvectors of $\mathcal{L}$ as

$$
\mathcal{I}=\bigoplus_{\mu} \sum_{i} \frac{\left.\left|\tau_{i}^{\mu}\right\rangle\right\rangle\left\langle\left\langle\bar{\tau}_{i}^{\mu}\right|\right.}{\left\langle\left\langle\bar{\tau}_{i}^{\mu} \mid \tau_{i}^{\mu}\right\rangle\right\rangle}
$$

\section{a. $\mathcal{L}_{a}$}

The eigenvalues and the right eigenvectors of $\mathcal{L}_{a}$ are

$$
\begin{aligned}
\left.\left|\tau_{1}^{a}\right\rangle\right\rangle= & |G G\rangle\rangle, \quad \lambda_{1}^{a}=0, \\
\left.\left|\tau_{2}^{a}\right\rangle\right\rangle= & \left.\left.\left.|E E\rangle\rangle-x_{1}\left|S_{R} S_{R}\right\rangle\right\rangle-x_{2}\left|A_{R} A_{R}\right\rangle\right\rangle-z\left|S_{R} A_{R}\right\rangle\right\rangle \\
& \left.\left.-z^{*}\left|A_{R} S_{R}\right\rangle\right\rangle+y|G G\rangle\right\rangle, \quad \lambda_{2}^{a}=-2 \gamma_{0}, \\
\left.\left|\tau_{3}^{a}\right\rangle\right\rangle= & \left.\frac{\left.\left|S_{R} A_{R}\right\rangle\right\rangle}{\left\langle A_{R} \mid S_{R}\right\rangle}-|G G\rangle\right\rangle, \quad \lambda_{3}^{a}=-\gamma_{0}-i V_{i}, \\
\left.\left|\tau_{4}^{a}\right\rangle\right\rangle= & \left.\frac{\left.\left|A_{R} S_{R}\right\rangle\right\rangle}{\left\langle S_{R} \mid A_{R}\right\rangle}-|G G\rangle\right\rangle, \quad \lambda_{4}^{a}=-\gamma_{0}+i V_{i}, \\
\left.\left|\tau_{5}^{a}\right\rangle\right\rangle= & \left.\left.\left|S_{R} S_{R}\right\rangle\right\rangle-|G G\rangle\right\rangle, \quad \lambda_{5}^{a}=-\gamma_{0}-V_{r}, \\
\left.\left|\tau_{6}^{a}\right\rangle\right\rangle= & \left.\left.\left|A_{R} A_{R}\right\rangle\right\rangle-|G G\rangle\right\rangle, \quad \lambda_{6}^{a}=-\gamma_{0}+V_{r},
\end{aligned}
$$

where $\left.\left|S_{R} S_{R}\right\rangle\right\rangle$ and $\left.\left|A_{R} A_{R}\right\rangle\right\rangle$ are the projectors of the kets $\left|A_{R}\right\rangle$ and $\left|S_{R}\right\rangle$, defined in Eq. (6), and where $x_{1}, x_{2}, y$, and $z$ have a complicated dependence on the various parameters of the model, not reported here. We remark that the eigenvector $\left.\left.\left|\tau_{1}^{a}\right\rangle\right\rangle=|G G\rangle\right\rangle$ associated with the eigenvalue $\lambda_{1}^{a}=0$ is the steady state of the two-atom system. The left eigenvectors in the sector $\mathcal{L}_{a}$ are (we report the right eigenvectors of $\mathcal{L}_{a}^{\dagger}$ )

$$
\begin{aligned}
\left.\left|\bar{\tau}_{1}^{a}\right\rangle\right\rangle & =|\mathbb{I}\rangle\rangle, \\
\left.\left|\bar{\tau}_{2}^{a}\right\rangle\right\rangle & =|E E\rangle\rangle, \\
\left.\left|\bar{\tau}_{3}^{a}\right\rangle\right\rangle & \left.=\frac{\left.\left|S_{L} A_{L}\right\rangle\right\rangle}{\operatorname{Tr}\left(\left|S_{L}\right\rangle\left\langle A_{L}\right|\right)}+x|E E\rangle\right\rangle, \\
\left.\left|\bar{\tau}_{4}^{a}\right\rangle\right\rangle & \left.=\frac{\left.\left|A_{L} S_{L}\right\rangle\right\rangle}{\operatorname{Tr}\left(\left|A_{L}\right\rangle\left\langle S_{L}\right|\right)}+x^{*}|E E\rangle\right\rangle, \\
\left.\left|\bar{\tau}_{5}^{a}\right\rangle\right\rangle & \left.\left.=\left|S_{L} S_{L}\right\rangle\right\rangle+y_{1}|E E\rangle\right\rangle, \\
\left.\left|\bar{\tau}_{6}^{a}\right\rangle\right\rangle & \left.\left.=\left|A_{L} A_{L}\right\rangle\right\rangle+y_{2}|E E\rangle\right\rangle,
\end{aligned}
$$

where $|\mathbb{I}\rangle\rangle$ is the vector associated with the identity in the four-dimensional space (in the decoupled basis it is $|\mathbb{I}\rangle\rangle=$ $|E E\rangle\rangle+|e g e g\rangle\rangle+|\operatorname{geg} e\rangle\rangle+|G G\rangle\rangle)$ and where

$$
\begin{gathered}
y_{1}=\frac{\gamma_{0}+V_{r}+\left(\gamma_{1}-\gamma_{2}\right) \frac{1-\left|\alpha_{S}\right|^{2}}{1+\left|\alpha_{S}\right|^{2}}}{\gamma_{0}-V_{r}}, \\
y_{2}=\frac{\gamma_{0}-V_{r}+\left(\gamma_{1}-\gamma_{2}\right) \frac{1-\left|\alpha_{A}\right|^{2}}{1+\left|\alpha_{A}\right|^{2}}}{\gamma_{0}+V_{r}}, \\
x=\left|\alpha_{A}\right| \frac{\gamma_{1}+\gamma_{2} \alpha_{A} \alpha_{S}^{*}+\gamma_{12}\left(\alpha_{A}+\alpha_{S}^{*}\right)}{\left(1+\left|\alpha_{A}\right|^{2}\right)\left\langle A_{L} \mid S_{L}\right\rangle\left(\gamma_{0}+i V_{i}\right)} . \\
\text { b. } \mathcal{L}_{b}, \mathcal{L}_{c}, \mathcal{L}_{d} \text { and } \mathcal{L}_{c}
\end{gathered}
$$

The eigenvalues of $\mathcal{L}_{b}$ are

$$
\begin{aligned}
& \lambda_{1}^{b}=-\frac{1}{2}\left[3 \gamma_{0}+V^{*}\right]-i \omega_{0}, \\
& \lambda_{2}^{b}=-\frac{1}{2}\left[3 \gamma_{0}-V^{*}\right]-i \omega_{0}, \\
& \lambda_{3}^{b}=-\frac{1}{2}\left(\gamma_{0}+V\right)-i \omega_{0}, \\
& \lambda_{4}^{b}=-\frac{1}{2}\left(\gamma_{0}-V\right)-i \omega_{0} .
\end{aligned}
$$

Concerning the right and left eigenvectors of $\mathcal{L}_{b}$, we found the analytical expressions for $\left.\left|\tau_{3,4}^{b}\right\rangle\right\rangle$ and $\left.\left|\bar{\tau}_{1,2}^{b}\right\rangle\right\rangle$ (we report the right eigenvectors of $\mathcal{L}_{b}^{\dagger}$ ):

$$
\left.\left.\left.\left.\left|\tau_{3}^{b}\right\rangle\right\rangle=\left|S_{R} G\right\rangle\right\rangle, \quad\left|\tau_{4}^{b}\right\rangle\right\rangle=\left|A_{R} G\right\rangle\right\rangle
$$


and

$$
\left.\left.\left.\left.\left|\bar{\tau}_{1}^{b}\right\rangle\right\rangle=\left|E S_{L}\right\rangle\right\rangle, \quad\left|\bar{\tau}_{2}^{b}\right\rangle\right\rangle=\left|E A_{L}\right\rangle\right\rangle
$$

The expressions for the other eigenvectors are particularly cumbersome and are then not reported here. The eigenvalues in the sector $\mathcal{L}_{c}$ are the conjugates of the ones in $\mathcal{L}_{b}$, while the right and left eigenvectors are given by $\tau_{i}^{c}=\tau_{i}^{b \dagger}$ and $\bar{\tau}_{i}^{c}=\bar{\tau}_{i}^{b \dagger}$. The only eigenvalue in $\mathcal{L}_{d}$ is equal to $\lambda_{1}^{d}=-\gamma_{0}-2 i \omega_{0}$ and the one in $\mathcal{L}_{e}$ is $\lambda_{1}^{e}=\lambda_{1}^{d *}$. The corresponding right and left eigenvectors are $\left.\left.\left.\left|\tau_{1}^{d}\right\rangle\right\rangle=\left|\bar{\tau}_{1}^{d}\right\rangle\right\rangle=|E G\rangle\right\rangle$ and $\left.\left.\left|\tau_{1}^{e}\right\rangle\right\rangle=\left|\bar{\tau}_{1}^{e}\right\rangle\right\rangle=$ $|G E\rangle\rangle$.

\section{Rate equations}

Here we report the equations of motion for the density-matrix elements belonging to the sector $\mathcal{L}_{a}$. In the decoupled basis the rate equations for the populations and the coherences between $|e g\rangle$ and $|g e\rangle$ can be immediately derived using the form of $\mathcal{L}_{a}$ of Eq. (A4) (we use the notation $\langle I|\rho| J\rangle=\rho_{I, J}$ ). In this basis, the populations of $|e g\rangle$ and $|g e\rangle$ are coupled with their coherences. The special role played by the states $\left|S_{L}\right\rangle$ and $\left|A_{L}\right\rangle$ emerges clearly when we derive the equations of motion for their populations, together with the equations of motion for their coherences, and for the ground state. These can be obtained by recombining the rate equations in the decoupled basis, exploiting Eq. (6), or, equivalently, by using the left eigenvectors of Eq. (A9) and the relation, for an arbitrary operator $O, \frac{d\langle O\rangle}{d t}=\left[\operatorname{Tr}\left(\rho \mathcal{L}^{\dagger} O^{\dagger}\right)\right]^{*}$ :

$$
\begin{aligned}
\frac{d \rho_{E, E}}{d t}= & -\left(\gamma_{1}+\gamma_{2}\right) \rho_{E, E}, \\
\frac{d \rho_{S_{L}, S_{L}}}{d t}= & {\left[\gamma_{0}+V_{r}-\left(\gamma_{1}-\gamma_{2}\right) \frac{1-\left|\alpha_{A}\right|^{2}}{1+\left|\alpha_{A}\right|^{2}}\right] \rho_{E, E}-\left(\gamma_{0}+V_{r}\right) \rho_{S_{L}, S_{L}}, } \\
\frac{d \rho_{S_{L}, A_{L}}}{d t}= & \left|\alpha_{A}\right| \frac{\gamma_{1}+\gamma_{2} \alpha_{S} \alpha_{A}^{*}+\gamma_{12}\left(\alpha_{S}+\alpha_{A}^{*}\right)}{\left(1+\left|\alpha_{A}\right|^{2}\right)} \rho_{E, E}-\left(\gamma_{0}+i V_{i}\right) \rho_{S_{L}, A_{L}}, \\
\frac{d \rho_{A_{L}, S_{L}}}{d t}= & \left|\alpha_{A}\right| \frac{\gamma_{1}+\gamma_{2} \alpha_{A} \alpha_{S}^{*}+\gamma_{12}\left(\alpha_{A}+\alpha_{S}^{*}\right)}{\left(1+\left|\alpha_{A}\right|^{2}\right)} \rho_{E, E}-\left(\gamma_{0}-i V_{i}\right) \rho_{A_{L}, S_{L}}, \\
\frac{d \rho_{A_{L}, A_{L}}}{d t}= & {\left[\gamma_{0}-V_{r}+\left(\gamma_{1}-\gamma_{2}\right) \frac{1-\left|\alpha_{A}\right|^{2}}{1+\left|\alpha_{A}\right|^{2}}\right] \rho_{E, E}-\left(\gamma_{0}-V_{r}\right) \rho_{A_{L}, A_{L}}, } \\
\frac{d \rho_{G, G}}{d t}= & \frac{1+\left|\alpha_{A}\right|^{2}}{\left|1+\alpha_{A}^{2}\right|^{2}}\left\{\left(1+\left|\alpha_{A}\right|^{2}\right)\left(\gamma_{0}+V_{r}\right) \rho_{S_{L}, S_{L}}+\left[-\gamma_{1}\left|\alpha_{A}\right|+\gamma_{2} \frac{\alpha_{A}^{* 2}}{\left|\alpha_{A}\right|}+\gamma_{12}\left(\alpha_{A}\left|\alpha_{A}\right|-\frac{\alpha_{A}}{\left|\alpha_{A}\right|}\right)\right] \rho_{S_{L}, A_{L}}\right. \\
& \left.+\left[-\gamma_{1}\left|\alpha_{A}\right|+\gamma_{2} \frac{\alpha_{A}^{2}}{\left|\alpha_{A}\right|}+\gamma_{12}\left(\alpha_{A}^{*}\left|\alpha_{A}\right|-\frac{\alpha_{A}^{*}}{\left|\alpha_{A}\right|}\right)\right] \rho_{A_{L}, S_{L}}+\left(1+\left|\alpha_{A}\right|^{2}\right)\left(\gamma_{0}-V_{r}\right) \rho_{A_{L}, A_{L}}\right\} .
\end{aligned}
$$

We remark that, in Eqs. (A14) and as depicted in Fig. 1, $\rho_{S_{L}, S_{L}}, \rho_{S_{L}, A_{L}}, \rho_{A_{L}, S_{L}}$, and $\rho_{A_{L}, A_{L}}$ are, respectively, coupled only to themselves and $\rho_{E, E}$.

[1] M. Gross and S. Haroche, Phys. Rep. 93, 301 (1982).

[2] A. Pikovsky, M. Rosenblum, and J. Kurths, Synchronization: A Universal Concept in Nonlinear Sciences (Cambridge University Press, Cambridge, 2001).

[3] R. H. Dicke, Phys. Rev. 93, 99 (1954).

[4] B. M. Garraway, Philos. Trans. R. Soc. London A 369, 1137 (2011).

[5] R. G. DeVoe and R. G. Brewer, Phys. Rev. Lett. 76, 2049 (1996).

[6] J. Eschner, C. Raab, F. Schmidt-Kaler, and R. Blatt, Nature (London) 413, 495 (2001).

[7] A. F. van Loo, A. Fedorov, K. Lalumière, B. C. Sanders, A. Blais, and A. Wallraff, Science 342, 1494 (2013).

[8] C. W. Chou, S. V. Polyakov, A. Kuzmich, and H. J. Kimble, Phys. Rev. Lett. 92, 213601 (2004); A. T. Black, J. K. Thompson, and V. Vuletic, ibid. 95, 133601 (2005).

[9] L.-M. Duan, M. D. Lukin, J. I. Cirac, and P. Zoller, Nature (London) 414, 413 (2001); R. Reimann, W. Alt, T. Kampschulte,
T. Macha, L. Ratschbacher, N. Thau, S. Yoon, and D. Meschede, Phys. Rev. Lett. 114, 023601 (2015).

[10] H. W. Chan, A. T. Black, and V. Vuletic, Phys. Rev. Lett. 90, 063003 (2003); M. Wolke, J. Klinner, H. Keßler, and A. Hemmerich, Science 337, 75 (2012).

[11] J. A. Mlynek, A. A. Abdumalikov, C. Eichler, and A. Wallraff, Nat. Commun. 5, 5186 (2014).

[12] A. Goban, C.-L. Hung, J. D. Hood, S.-P. Yu, J. A. Muniz, O. Painter, and H. J. Kimble, Phys. Rev. Lett. 115, 063601 (2015).

[13] W. Guerin, M. O. Araújo, and R. Kaiser, Phys. Rev. Lett. 116, 083601 (2016).

[14] G. L. Giorgi, F. Galve, G. Manzano, P. Colet, and R. Zambrini, Phys. Rev. A 85, 052101 (2012); G. Manzano, F. Galve, G. L. Giorgi, E. Hernandez-Garcia, and R. Zambrini, Sci. Rep. 3, 1439 (2013).

[15] G. Heinrich, M. Ludwig, J. Qian, B. Kubala, and F. Marquardt, Phys. Rev. Lett. 107, 043603 (2011); M. Ludwig and F. 
Marquardt, ibid. 111, 073603 (2013); A. Mari, A. Farace, N. Didier, V. Giovannetti, and R. Fazio, ibid. 111, 103605 (2013).

[16] H. B. Qiu, B. Juliá-Díaz, M. A. García-March, and A. Polls, Phys. Rev. A 90, 033603 (2014); H. Qiu, R. Zambrini, A. Polls, J. Martorell, and B. Juliá-Díaz, ibid. 92, 043619 (2015).

[17] M. R. Hush, W. Li, S. Genway, I. Lesanovsky, and A. D. Armour, Phys. Rev. A 91, 061401 (2015).

[18] G. L. Giorgi, F. Plastina, G. Francica, and R. Zambrini, Phys. Rev. A 88, 042115 (2013).

[19] B. Zhu, J. Schachenmayer, M. Xu, F. Herrera, J. G. Restrepo, M. J. Holland, and A. M. Rey, New J. Phys. 17, 083063 (2015).

[20] G. L. Giorgi, F. Galve, and R. Zambrini, Phys. Rev. A 94, 052121 (2016).

[21] F. Galve, G. L. Giorgi, and R. Zambrini, arXiv:1610.05060.

[22] I. Goychuk, J. Casado-Pascual, M. Morillo, J. Lehmann, and P. Hänggi, Phys. Rev. Lett. 97, 210601 (2006); O. V. Zhirov and D. L. Shepelyansky, ibid. 100, 014101 (2008); S. B. Shim, M. Imboden, and P. Mohanty, Science 316, 95 (2007).

[23] D. Meiser, J. Ye, D. R. Carlson, and M. J. Holland, Phys. Rev. Lett. 102, 163601 (2009); D. Meiser and M. J. Holland, Phys. Rev. A 81, 033847 (2010).

[24] J. G. Bohnet, Z. Chen, J. M. Weiner, D. Meiser, M. J. Holland, and J. K. Thompson, Nature (London) 484, 78 (2012).

[25] M. Xu, D. A. Tieri, E. C. Fine, J. K. Thompson, and M. J. Holland, Phys. Rev. Lett. 113, 154101 (2014).
[26] G. M. Palma, A. Vaglica, C. Leonardi, F. A. M. DeOliveira, and P. L. Knight, Opt. Commun. 79, 377 (1990).

[27] G. M. Palma, A. Vaglica, C. Leonardi, and A. K. Ekert, Opt. Commun. 86, 192 (1991).

[28] C. Leonardi and A. Vaglica, J. Phys. B 14, L307 (1981); Opt. Commun. 53, 340 (1985).

[29] Z. Ficek, R. Tanaś, and S. Kielich, Physica A 146, 452 (1987).

[30] G. M. Palma and P. L. Knight, Phys. Rev. A 39, 1962 (1989); A. K. Ekert, G. M. Palma, S. M. Barnett, and P. L. Knight, ibid. 39, 6026 (1989).

[31] Z. Ficek and R. Tanaś, Phys. Rep. 372, 369 (2002).

[32] G. S. Agarwal, in Quantum Statistical Theories of Spontaneous Emission and their Relation to other Approaches, edited by G. Höhler, Springer Tracts in Modern Physics Vol. 70 (Springer, Berlin, 1974), pp. 1-128.

[33] Z. Ficek and S. Swain, Quantum Interference and Coherence: Theory and Experiments (Springer, New York, 2005).

[34] H.-P. Breuer and F. Petruccione, The Theory of Open Quantum Systems (Oxford University Press, New York, 2002).

[35] R. H. Lehmberg, Phys. Rev. A 2, 883 (1970).

[36] F. Galve, A. Mandarino, M. G. A. Paris, C. Benedetti, and R. Zambrini, Sci. Rep. 7, 42050 (2017).

[37] G. M. Palma, K.-A. Suominen, and A. K. Ekert, Proc. R. Soc. London A 452, 567 (1996).

[38] M. Ban, Eur. Phys. J. D 58, 415 (2010). 